\title{
Abdominal obesity in type 1 diabetes associated with gender, cardiovascular risk factors and complications, and difficulties achieving treatment targets: a cross sectional study at a secondary care diabetes clinic
}

Eva O. Melin ${ }^{1,2,3^{*}}$, Hans O. Thulesius ${ }^{2,3,4}$, Magnus Hillman' ${ }^{5}$ Mona Landin-Olsson ${ }^{1,5,6}$ and Maria Thunander ${ }^{1,2,7}$

\begin{abstract}
Background: Abdominal obesity is linked to cardiovascular diseases in type 1 diabetes (T1D). The primary aim was to explore associations between abdominal obesity and cardiovascular complications, metabolic and inflammatory factors. The secondary aim was to explore whether achieved recommended treatment targets differed between the obese and non-obese participants.

Methods: Cross sectional study of 284 T1D patients (age 18-59 years, men 56\%), consecutively recruited from one secondary care specialist diabetes clinic in Sweden. Anthropometrics, blood pressure, serum-lipids and highsensitivity C-reactive protein (hs-CRP) were collected and supplemented with data from the patients' medical records and from the Swedish National Diabetes Registry. Abdominal obesity was defined as waist circumference men/women (meters): $\geq 1.02 / \geq 0.88$. Hs-CRP was divided into low-, moderate-, and high-risk groups for future cardiovascular events $(<1,1$ to 3 , and $>3$ to $\leq 8.9 \mathrm{mg} / \mathrm{l})$. Treatment targets were blood pressure $\leq 130 / \leq 80$, total cholesterol $\leq 4.5 \mathrm{mmol} / \mathrm{l}, \mathrm{LDL}: \leq 2.5 \mathrm{mmol} / \mathrm{l}$, and HbA1c: $\leq 52 \mathrm{mmol} / \mathrm{mol}(\leq 6.9 \%)$. Different explanatory linear, logistic and ordinal regression models were elaborated for the associations, and calibrated and validated for goodness of fit with the data variables.

Results: The prevalence of abdominal obesity was 49/284 (17\%), men/women: 8\%/29\% ( $P<0.001)$. Women (adjusted odds ratio (AOR) 6.5), cardiovascular complications (AOR 5.7), HbA1c > 70 mmol/mol (> 8.6\%) (AOR 2.7), systolic blood pressure (per $\mathrm{mm} \mathrm{Hg}$ ) (AOR 1.05), and triglycerides (per mmol/I) (AOR 1.7), were associated with abdominal obesity. Sub analyses ( $n=171$ ), showed that abdominal obesity (AOR 5.3) and triglycerides (per mmol/I) (AOR 2.8) were associated with increasing risk levels of hs-CRP. Treatment targets were obtained for fewer patients with abdominal obesity for $\mathrm{HbA} 1 \mathrm{c}(8 \%$ vs $21 \%, P=0.044)$ and systolic blood pressure ( $51 \%$ vs $68 \%, P=0.033)$. No patients with abdominal obesity reached all treatment targets compared to $8 \%$ in patients without abdominal obesity.

(Continued on next page)
\end{abstract}

\footnotetext{
* Correspondence: eva.melin@kronoberg.se; eva.o.melin@gmail.com

1 Department of Clinical Sciences, Section Endocrinology and Diabetes, Lund University, Lund, Sweden

2Department of Research and Development, Region Kronoberg, Box 1223,

SE-35112 Växjö, Sweden

Full list of author information is available at the end of the article
}

(c) The Author(s). 2018 Open Access This article is distributed under the terms of the Creative Commons Attribution 4.0 International License (http://creativecommons.org/licenses/by/4.0/), which permits unrestricted use, distribution, and reproduction in any medium, provided you give appropriate credit to the original author(s) and the source, provide a link to the Creative Commons license, and indicate if changes were made. The Creative Commons Public Domain Dedication waiver (http://creativecommons.org/publicdomain/zero/1.0/) applies to the data made available in this article, unless otherwise stated. 
(Continued from previous page)

Conclusions: Significant associations between abdominal obesity and gender, cardiovascular disease, and the cardiovascular risk factors low-grade inflammation, systolic blood pressure, high HbA1c, and triglycerides, were found in 284 T1D patients. Fewer patients with abdominal obesity reached the treatment targets for HbA1c and systolic blood pressure compared to the non-obese.

Keywords: Abdominal obesity, Cardiovascular complications, Diabetes mellitus type 1, Gender, Glycemic control, Hyperlipidemia, Hypertension, Inflammation, Treatment targets

\section{Background}

Both women and men with type 1 diabetes mellitus (T1D) have increased cardiovascular and all-cause mortality compared to persons without T1D, and the risk for premature death is increasing with increasing $\mathrm{HbA1c}$ levels [1]. Women with T1D are described to be at particular risk for both coronary artery calcification and for cardiovascular death across all age groups $[1,2]$. The introduction of intensified insulin therapy for patients with type 1 diabetes mellitus (T1D) has led to decreased prevalence of diabetic retinopathy, nephropathy and neuropathy [3]. Intensive insulin therapy has however two major side effects, weight gain and increased frequency of severe episodes of hypoglycaemia [3]. Excess weight gain in T1D is associated with abdominal obesity, insulin resistance, dyslipidaemia, higher blood pressure, and atherosclerosis [4]. Particularly girls/women with T1D are at risk for developing overweight and obesity [5]. The prevalence of obesity is increasing globally [6]. When this study was conducted in 2009, the prevalence of general obesity (BMI $\geq 30 \mathrm{~kg} / \mathrm{m}^{2}$ ) was $11 \%$ in men, and $10 \%$ in women in the general population in Sweden [7].

There is evidence that low-density lipoprotein (LDL) is both an indicator of future cardiovascular risk and a causal agent in the atherothrombotic process [8]. Raised triglycerides have been associated with low-grade inflammation, artery calcification, cardiovascular disease and all-cause mortality, and there is evidence that triglycerides are causal in the atherosclerosis process [9-12]. Common causes of raised triglycerides are obesity and high alcohol intake [9]. Impaired glycemic control has been linked to raised triglycerides in type 2 diabetes (T2D) [9]. Low levels of high-density lipoprotein (HDL) are strong predictors of atherosclerosis and cardiovascular disease [13]. However the causal relation between HDL and atherosclerosis is uncertain [13]. Lipidlowering drugs are associated with a reduced risk of cardiovascular disease and death in T1D [14].

Chronic low-grade inflammation has been associated with obesity, insulin resistance, hypertension, hyperglycemia, acute hypoglycemia, dyslipidaemia, cardiovascular disease, and smoking [15-18]. One of the most frequently used markers of low-grade inflammation is high sensitivity C-reactive protein (hs-CRP), which is atherogenic, and a strong predictor of future cardiovascular events $[15,19]$. Hs-CRP might be involved in mediating atherothrombotic disease through activation of complement pathways and immune cells [20].

In line with both international and Swedish national guidelines for diabetes, indications for lipid-lowering drugs at the clinic in 2009 were total cholesterol (TC) > $4.5 \mathrm{mmol} / \mathrm{l}$ or LDL $>2.5 \mathrm{mmol} / \mathrm{l}$, in addition to dietary interventions and increased physical activity [21-23]. Indications for anti-hypertensive drugs were systolic blood pressure $>130 \mathrm{mmHg}$, or diastolic blood pressure > $80 \mathrm{mmHg}[18,21-24]$.

We have recently found that alexithymia, which is characterized by impaired capacity to identify and describe feelings, was associated with abdominal obesity [25]. We have also previously found that abdominal obesity, depression and smoking were independently associated with inadequate glycemic control [26].

The primary aim was to explore links between abdominal obesity, metabolic and inflammatory factors and cardiovascular complications in persons with T1D. The secondary goal was to explore whether obtained treatment targets for $\mathrm{HbA1c}$, blood pressure, $\mathrm{TC}$ and $\mathrm{LDL}$ differed between the obese and non-obese participants.

\section{Methods \\ Participants and procedures}

This study had a cross sectional design and was one of four baseline analyses [25-27] for a randomized controlled trial (ClinicalTrials.gov: NCT01714986) where "Affect School with Script Analysis" was tried against "Basic Body Awareness Therapy" for persons with diabetes, inadequate glycemic control and psychological symptoms $[28,29]$. The participants were outpatients, consecutively recruited by specialist diabetes physicians or diabetes nurses, at regular follow up visits during the period $03 / 25 / 2009$ to $12 / 28 / 2009$. They were recruited from one secondary care specialist diabetes clinic, with a catchment population of 125,000 in southern Sweden. In this study 284 persons with T1D were included, $66 \%$ of the eligible patients (Fig. 1). Exclusion criteria were cancer, hepatic failure, end-stage renal disease, stroke with cognitive deficiency, psychotic disorder, bipolar disorder, 


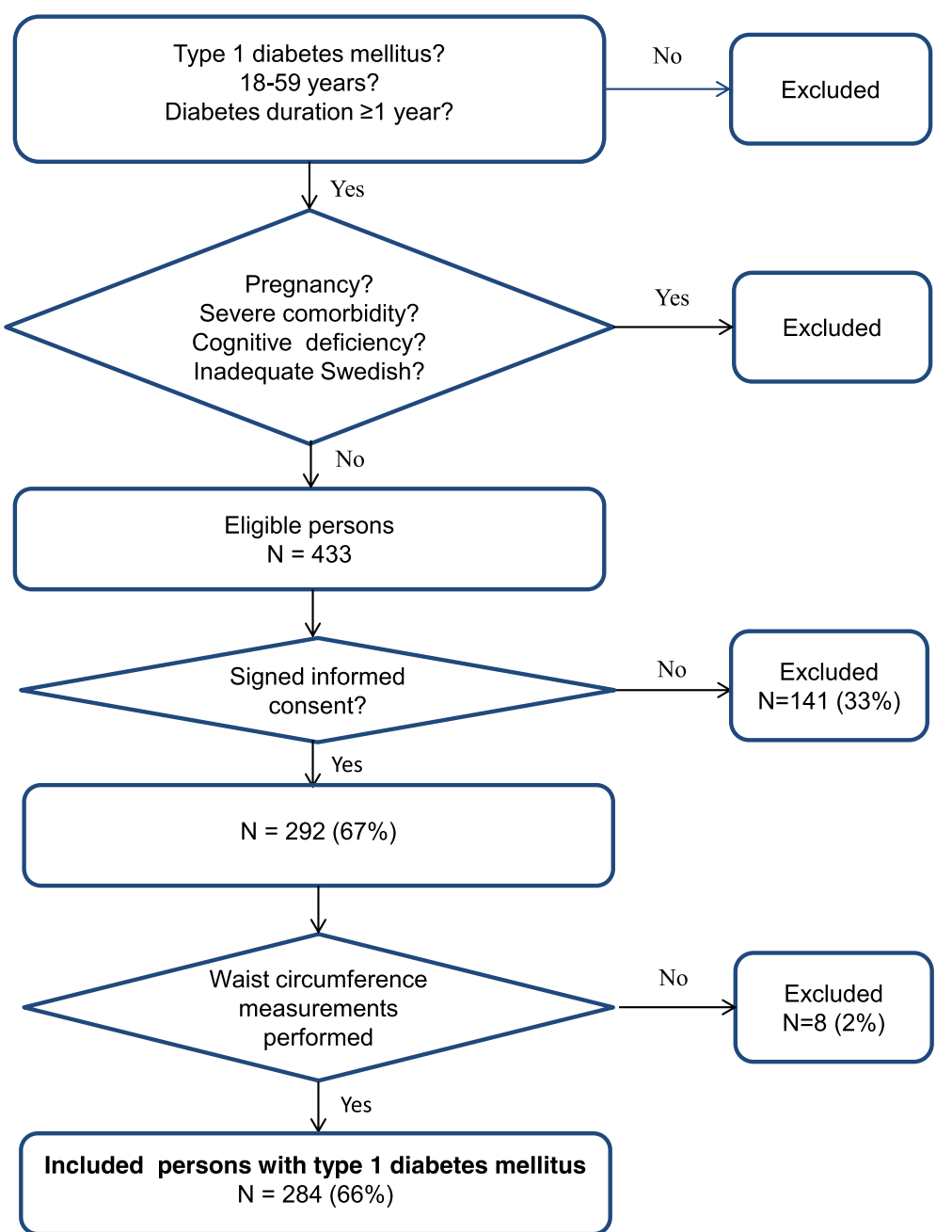

Fig. 1 Description of criteria for inclusion in the study of obesity in persons with T1D

severe personality disorder, severe substance abuse, or mental retardation. Anthropometrics, blood pressure and blood samples were collected. Data were collected from computerized medical records and the Swedish national diabetes register (S-NDR) $[1,23]$.

\section{Medication}

Diabetes specific treatment was divided into three groups: multiple daily insulin injections (MDII), continuous subcutaneous insulin infusion (CSII), and MDII combined with oral antidiabetic agents (OAA) (ATC code A10BA02). The indications for OAA prescription in addition to insulin were obesity and insulin resistance.

Anti-hypertensive drugs were calcium antagonists (ATC codes C08CA01-02); angiotensin-converting enzyme (ACE) inhibitors (ATC codes C09AA-BA), angiotensin II antagonists (ATC codes C09CA-DA; diuretics (ATC code C03A); selective beta-adrenoreceptor antagonists (ATC code C07AB).
Lipid-lowering drugs were HMG CoA-reductase inhibitors (statins) (C10AA).

\section{Anthropometrics and blood pressure}

Waist circumference (WC), weight, length and blood pressure were measured according to standard procedures by a nurse. Abdominal obesity was defined as WC men/ women (meters): $\geq 1.02 />0.88$ [30-32]. General obesity was defined as Body Mass Index (BMI): $\geq 30 \mathrm{~kg} / \mathrm{m}^{2}$ for both genders [31].

\section{HbA1C, serum-lipids and hs-CRP}

$\mathrm{HbA} 1 \mathrm{c}$ and serum lipids analyses were performed at the department of Clinical Chemistry, Växjö Central Hospital.

Venous HbA1c was analyzed with high pressure liquid chromatography, HPLC - variant II, Turbo analyzer (Bio - Rad $^{\circ}$, Hercules, CA, USA). HbA1c $>70 \mathrm{mmol} / \mathrm{mol}$ (> $8.6 \%$ ) corresponds to the 75 th percentile in the whole population sample [26]. 
After an overnight fast, blood samples were collected and serum-lipids were were measured directly [8], using the enzymatic colour test (Olympus $\mathrm{AU}^{\oplus}$, Tokyo, Japan). High TC was defined as $>4.5 \mathrm{mmol} / \mathrm{l}$, high LDL as > $2.5 \mathrm{mmol} / \mathrm{l}$, high triglycerides as $\geq 1.7 \mathrm{mmol} / \mathrm{l}$; low HDL as $<1.04 \mathrm{mmol} / \mathrm{l}$ for men, and as $<1.29 \mathrm{mmol} / \mathrm{l}$ for women [33].

Samples for hs-CRP were collected, centrifuged, and stored at - $70 \mathrm{C}$ Celsius until analyzed with spectrophotometry on a Roche Cobas C501 at the diabetes laboratory, Lund University Hospital, Lund. Hs-CRP was $0.54 \pm$ $0.02 \mathrm{mg} / \mathrm{l}$ in healthy subjects according to previous research [16]. Hs-CRP $<1,1$ to 3 , and $>3$ to $\leq 10 \mathrm{mg} / \mathrm{l}$ correspond to low-, moderate- and high-risk groups for future cardiovascular events [19]. Samples with hs-CRP $\geq 10 \mathrm{mg} / \mathrm{l}$ were excluded as recommended in previous research [19]. Samples stored $>1$ year were excluded. HsCRP was available for 171 (60\%) participants.

\section{Treatment targets according to the Swedish National Guidelines for diabetes in 2009}

The treatment targets recommend by the Swedish National Board of Health and Welfare were for T1D patients: 1) glycemic control: HbA1c $\leq 52 \mathrm{mmol} / \mathrm{mol}$; 2) systolic/diastolic blood pressure: $\leq 130 / \leq 80 \mathrm{mmHg}$; 3 ) serum-lipids: $\mathrm{TC} \leq 4.5$ and $\mathrm{LDL} \leq 2.5 \mathrm{mmol} / \mathrm{l}[22]$.

\section{Hypoglycemia episodes}

A severe hypoglycemia episode was defined as needing help from another person. Episodes during the last 6 months prior to recruitment were registered.

\section{Smoking and physical inactivity}

Smokers were defined as having smoked any amount of tobacco during the last year.

Physical inactivity was defined as moderate activities, such as 30 min of walking, less than once a week.

\section{Cardiovascular complications}

Cardiovascular complications were defined as ischemic heart disease or stroke/TIA.

\section{Statistical analysis}

Analysis of data distribution using histograms revealed that age, diabetes duration, hs-CRP, triglycerides, BMI and WC were not normally distributed. Data were presented as median values (quartile $(\mathrm{q})_{1}, \mathrm{q}_{3}$; range), and analyses were performed with Mann-Whitney $U$ test. Fisher's exact test (two-tailed) and Linear-by-Linear Association (two-tailed) were used to analyze categorical data. Crude odds ratios (CORs) were calculated, variables with $P \leq 0.10$, and age independent of $P$-value, were entered in multiple logistic regression analyses (Backward: Wald). The Hosmer and Lemeshow test for goodness-of- fit and Nagelkerke $\mathrm{R}^{2}$ were used to evaluate each multiple logistic regression analysis model. Ordinal regression analysis (stepwise forward) was performed with 3 risk levels of hs-CRP as dependent variables. Variables with $P$ values $\leq 0.10$ in simple linear regression analyses were entered into multiple linear regression analyses (Backward). Confidence intervals (CIs) of $95 \%$ were used. $P<0.05$ was considered statistically significant. SPSS ${ }^{\bullet}$ version 18 (IBM, Chicago, Illinois, USA) was used for statistical analyses.

\section{Results}

In this population based cross sectional study of persons with T1D ( $n=284$, age $18-59$ years, men $56 \%)$, persons with abdominal obesity $(n=49)$ were compared with non-obese persons $(n=235)$. Baseline data including comparisons between men and women are presented in Table 1. The women, compared with the men, had higher prevalence of both abdominal obesity (29\% vs $8 \%, P<0.001)$ and general obesity $(18 \%$ vs $7 \%, P=0.005)$. The men had higher systolic and diastolic blood pressure (both $P<0.001$ ) and lower HDL $(P=0.002)$. The percentage that reached the recommended treatment targets were for HbA1c: $19 \%$; TC: 48\%; LDL: $36 \%$; systolic blood pressure: $65 \%$; diastolic blood pressure: 95\%. Only 7\% reached all treatment targets.

\section{Comparisons between patients with and without abdominal obesity}

Results of comparisons between 49 persons with abdominal obesity and 235 persons without abdominal obesity are presented in Table 2. Persons with abdominal obesity had higher prevalence of HbA1c $>70 \mathrm{mmol} / \mathrm{mol}(>8.6 \%)$ $(P<0.001)$, lipid-lowering drugs $(P=0.012)$ and cardiovascular complications $(P=0.016)$; and had higher median values of hs- CRP $(P<0.001)$, triglycerides $(P<0.001)$, systolic blood pressure $(P=0.004)$, LDL $(P=0.021)$ and TC $(P=0.047)$. Fewer patients with abdominal obesity compared to the non-obese reached the recommended treatment targets for HbA1c ( $8 \%$ vs $21 \%, P=0.044)$ and systolic blood pressure $(51 \%$ vs $68 \%, P=0.033)$. No patients with abdominal obesity reached all risk factor treatment targets for blood pressure, TC, LDL and HbA1c compared to $8 \%$ in the non-obese.

\section{Comparisons between users and non-users of anti- hypertensive and lipid-lowering drugs}

Persons treated with anti-hypertensive drugs had higher prevalence of high systolic blood pressure (62\% vs $22 \%$, $P<0.001)$, and cardiovascular complications $(P=0.018)$ (Table 2). Patients treated with lipid-lowering drugs had significantly higher median triglycerides $(P=0.014)$, higher prevalence of cardiovascular complications $(P=0$. 
Table 1 Baseline characteristics and comparisons between men and women for 284 persons with T1D

\begin{tabular}{|c|c|c|c|c|}
\hline & All patients & Men & Women & $p^{a}$ \\
\hline$N$ & 284 & $159(56)$ & $125(44)$ & \\
\hline Age (years) & $42(32,51 ; 18-59)$ & $43(32,52)$ & $41(30,50)$ & $0.12^{b}$ \\
\hline Diabetes duration (years) & $20(11,30 ; 1-55)$ & $21(11-32)$ & $19(11,29)$ & $0.26^{b}$ \\
\hline WC (meters) & - & $0.88(0.82,0.95 ; 0.65-1.33)$ & $0.79(0.75,0.90 ; 0.63-1.25)$ & - \\
\hline Abdominal obesity ${ }^{c}$ & $49(17)$ & $13(8)$ & $36(29)$ & $<0.001$ \\
\hline General obesity $^{d}$ & $34(12)$ & $11(7)$ & $23(18)$ & 0.005 \\
\hline $\mathrm{HbA1c}>52 \mathrm{mmol} / \mathrm{mol}(>6.9 \%)$ & $230(81)$ & $130(82)$ & $100(80)$ & 0.76 \\
\hline $\mathrm{HbA1c}>70 \mathrm{mmol} / \mathrm{mol}(>8.6 \%)$ & $39(24)$ & $39(24)$ & $39(31)$ & 0.23 \\
\hline $\mathrm{TC}(\mathrm{mmol} / \mathrm{l})$ & $4.6(4.1,5.2 ; 2.1-10.9)$ & $4.5(4.0,5.1)$ & $4.7(4.1,5.4)$ & $0.069^{b}$ \\
\hline High TC (> $4.5 \mathrm{mmol} / \mathrm{l})$ & $149(52)$ & $78(49)$ & $71(57)$ & 0.23 \\
\hline $\operatorname{LDL}(\mathrm{mmol} / \mathrm{l})$ & $2.8(2.4,3.3 ; 0.6-8.3)$ & $2.8(2.4,3.3)$ & $2.9(2.4,3.4)$ & $0.51^{b}$ \\
\hline High LDL (> $2.5 \mathrm{mmol} / \mathrm{l})$ & $182(64)$ & $102(64)$ & $80(64)$ & $>0.99$ \\
\hline Triglycerides $(\mathrm{mmol} / \mathrm{l})$ & $0.9(0.7,1.3 ; 0.6-5.9)$ & $0.9(0.7,1.3)$ & $0.8(0.6,1.3)$ & $0.47^{b}$ \\
\hline High triglycerides $(\geq 1.7 \mathrm{mmol} / \mathrm{l})$ & $34(12)$ & $16(10)$ & $18(14)$ & 0.28 \\
\hline $\mathrm{HDL}(\mathrm{mmol} / \mathrm{l})$ & $1.5(1.3,1.8 ; 0.8-2.7)$ & $1.4(1.2,1.7)$ & $1.6(1.4,1.8)$ & $0.002^{b}$ \\
\hline Low HDL (M/W: < 1.04/< $1.29 \mathrm{mmol} / \mathrm{l} /)$ & $32(11)$ & $17(11)$ & $15(12)$ & 0.85 \\
\hline $\mathrm{Hs}-\mathrm{CRP}^{\mathrm{e}}(\mathrm{mg} / \mathrm{l})$ & $0.6(0.3,1.7 ; 0.03-8.9)$ & $0.5(0.2,1.4)$ & $0.9(0.4,2.5)$ & $0.008^{b}$ \\
\hline $\mathrm{SBP}^{\mathrm{f}}(\mathrm{mm} \mathrm{Hg})$ & $120(111,130 ; 100-160)$ & $125(120,130)$ & $120(110,130)$ & $<0.001^{b}$ \\
\hline High SBP ${ }^{f}(>130 \mathrm{mmHg})$ & $100(35)$ & $67(42)$ & $33(26)$ & 0.006 \\
\hline $\mathrm{DBP}^{\mathrm{h}}(\mathrm{mm} \mathrm{Hg})$ & $70(70,75 ; 55-100)$ & $70(70,80)$ & $70(65,75)$ & $<0.001^{b}$ \\
\hline High DBP ${ }^{g}(>80$ mmHg) & $13(5)$ & $10(6)$ & $3(2)$ & 0.16 \\
\hline Hypoglycemia (severe episodes) & $13(5 \%)$ & $7(4)$ & $6(5)$ & $>0.99$ \\
\hline Smoking ${ }^{\mathrm{h}}$ & $28(10)$ & $18(12)$ & $10(8)$ & 0.42 \\
\hline Physical inactivity ${ }^{\mathrm{h}}(<1 /$ week $)$ & $31(11)$ & $18(12)$ & $13(11)$ & 0.85 \\
\hline CV ' complications & $10(4)$ & $6(4)$ & $4(3)$ & $>0.99$ \\
\hline $\operatorname{LLD}^{j}$ & $133(47)$ & $77(48)$ & $56(45)$ & 0.55 \\
\hline$A H D^{k}$ & $95(34)$ & $61(38)$ & $34(27)$ & 0.057 \\
\hline$M D \|^{\prime}$ and $O A D{ }^{m}$ & $17(6)$ & $6(4)$ & $11(9)$ & 0.15 \\
\hline $\operatorname{CS} \|^{n}$ & $26(9)$ & $13(8)$ & $13(10)$ & \\
\hline$M D \|^{\prime}$ & $241(85)$ & $140(88)$ & $101(81)$ & \\
\hline Reached all treatment targets ${ }^{\circ}$ & $19(7)$ & $11(7)$ & $8(6)$ & $>0.99$ \\
\hline
\end{tabular}

Data are $\mathrm{n}(\%)$ or median $\left(\mathrm{q}_{1}, \mathrm{q}_{3} ;\right.$ min-max)

${ }^{a}$ Fisher's exact test unless otherwise indicated. ${ }^{b}$ Mann-Whitney $U$ test. ${ }^{c}$ WC: men/women $\geq 1.02 / \geq 0.88 \mathrm{~m}$. ${ }^{\mathrm{d}} \mathrm{BMI} \geq 30 \mathrm{~kg} / \mathrm{m}^{2}$. ${ }^{\mathrm{e}} \mathrm{N}=171$, missing values for men/ women: $n=54 / 59$. ${ }^{\mathrm{f}}$ Systolic blood pressure. ${ }^{\mathrm{g}}$ Diastolic blood pressure. ${ }^{\mathrm{h}}$ Missing values men/women: $n=6 / 6$. ${ }^{\mathrm{i}}$ Cardiovascular. ${ }^{\mathrm{j}}$ Lipid-lowering drugs. ${ }^{\mathrm{k}}$ Antihypertensive drugs. ' Multiple daily insulin injections. ${ }^{\mathrm{m}}$ Oral antidiabetic drugs. ${ }^{\mathrm{n}}$ Continuous subcutaneous insulin infusion. ${ }^{\circ}$ Blood pressure $\leq 130 / \leq 80, \mathrm{TC} \leq 4.5$, $\mathrm{LDL} \leq 2.5$ and $\mathrm{HbA} 1 \mathrm{c} \leq 52$

007), and lower prevalence of high LDL $(P=0.006)$ than non-users of lipid-lowering drugs (Table 2).

\section{Factors associated with abdominal obesity}

Women (adjusted odds ratio (AOR) 6.5), systolic blood pressure (per mm Hg) (AOR 1.05), HbAlc > $70 \mathrm{mmol} /$ $\mathrm{mol}(>8.6 \%)$ (AOR 2.7), triglycerides (per mmol/l) (AOR 1.7), and cardiovascular complications (AOR 5.7) were associated with abdominal obesity (Table 3). Gender analyses showed that diastolic blood pressure (per $\mathrm{mm}$ $\mathrm{Hg}$ ) (AOR 1.13) and anti-hypertensive drugs (AOR 5.3) were associated with abdominal obesity in men. Triglycerides (per mmol/l) (AOR 2.1), lipid-lowering drugs (AOR 3.1), and HbA1c $>70 \mathrm{mmol} / \mathrm{mol}$ (> 8.6\%) (AOR 2. $9)$, were associated with abdominal obesity in women.

Factors associated with high-, moderate- and low-risk hsCRP levels in 171 persons

Abdominal obesity (AOR (CI) 5.3 (2.1-13.6)) and triglycerides (per mmol/l) (AOR (CI) 2.82 (1.68-4.93)) were associated with increasing risk levels of hs-CRP (Table 4). 
Table 2 Comparisons between obese and non-obese, users and non-users of antihypertensive and lipid-lowering drugs

\begin{tabular}{|c|c|c|c|c|c|c|c|c|c|}
\hline & \multicolumn{3}{|c|}{ Abdominal obesity } & \multicolumn{3}{|c|}{ Anti-hypertensive drugs } & \multicolumn{3}{|c|}{ Lipid-lowering drugs } \\
\hline & $\overline{Y e s}$ & No & $p^{a}$ & Yes & No & $p^{a}$ & $\overline{Y e s}$ & No & $p^{a}$ \\
\hline$N$ & $49(17)$ & $235(83)$ & & $95(33)$ & $189(67)$ & & $133(47)$ & $151(53)$ & \\
\hline Age & $45(35,53)$ & $42(31,51)$ & $0.11^{b}$ & $49(42,56)$ & $39(28,56)$ & $<0.001^{b}$ & $49(42,54)$ & $34(27,44)$ & $<0.001^{b}$ \\
\hline Diabetes duration & $22(14,28)$ & $20(11,31)$ & $0.64^{\mathrm{b}}$ & $29(20,35)$ & $16(9,25)$ & $<0.001^{b}$ & $26(14,34)$ & $17(9,24)$ & $<0.001^{b}$ \\
\hline Abdominal obesity & $49(100)$ & 0 & & $22(23)$ & $27(14)$ & 0.069 & $31(23)$ & $18(12)$ & 0.012 \\
\hline $\mathrm{HbA} 1 \mathrm{c}>52 \mathrm{mmol} / \mathrm{mol}(>6.9 \%)$ & $45(92)$ & $185(79)$ & 0.044 & $79(83)$ & $151(80)$ & 0.63 & $114(86)$ & $116(77)$ & 0.069 \\
\hline $\mathrm{HbA} 1 \mathrm{c}>70 \mathrm{mmol} / \mathrm{mol}(>8.6 \%)$ & $24(49)$ & $54(23)$ & $<0.001$ & $29(30)$ & $49(26)$ & 0.48 & $40(30)$ & $38(25)$ & 0.42 \\
\hline $\mathrm{TC}(\mathrm{mmol} / \mathrm{l})$ & $4.7(4.2,5.8)$ & $4.6(4.1,5.1)$ & $0.047^{b}$ & - & - & - & $4.5(4.0,5.2)$ & $4.6(4.1,5.2)$ & $0.32^{b}$ \\
\hline High TC (> $4.5 \mathrm{mmol} / \mathrm{l})$ & $29(59)$ & $120(51)$ & 0.35 & - & - & - & $63(47)$ & $86(57)$ & 0.12 \\
\hline Triglycerides (mmol/l) & $1.2(0.8,1.9)$ & $0.9(0.7,1.1)$ & $<0.001^{b}$ & - & - & - & $1.0(0.7,1.3)$ & $0.8(0.7,1.1)$ & $0.014^{b}$ \\
\hline High triglycerides ( $\geq 1.7 \mathrm{mmol} / \mathrm{l})$ & $14(29)$ & $20(8)$ & $<0.001$ & - & - & - & $21(16)$ & $13(9)$ & 0.070 \\
\hline $\mathrm{HDL}(\mathrm{mmol} / \mathrm{l})$ & $1.5(1.3,1.7)$ & $1.5(1.3,1.8)$ & $0.47^{b}$ & - & - & - & $1.5(1.3,1.8)$ & $1.5(1.3,1.8)$ & $0.48^{b}$ \\
\hline Low HDL (m/w: $<1.04 / 1.29 \mathrm{mmol} / \mathrm{l})$ & $5(10)$ & $27(11)$ & $>0.99$ & - & - & - & $12(9)$ & $20(13)$ & 0.35 \\
\hline LDL (mmol/l) & $3.2(2.5,3.8)$ & $2.8(2.4,3.3)$ & $0.021^{b}$ & - & - & - & $2.7(2.3,3.3)$ & $3.0(2.5,3.4)$ & $0.058^{b}$ \\
\hline High LDL (> $2.5 \mathrm{mmol} / \mathrm{l})$ & $36(74)$ & $146(62)$ & 0.14 & - & - & - & $74(56)$ & $108(72)$ & 0.006 \\
\hline $\mathrm{Hs}-\mathrm{CRP}^{\mathrm{c}}(\mathrm{mg} / \mathrm{l})$ & $2.5(0.6,4.6)$ & $0.6(0.2,1.4)$ & $<0.001^{b}$ & $0.6(0.3,1.7)$ & $0.7(0.3,1.9)$ & 0.87 & $0.6(0.3,1.8)$ & $0.8(0.3,1.7)$ & 0.97 \\
\hline $\mathrm{SBP}(\mathrm{mm} \mathrm{Hg})$ & $130(120,132)$ & $120(110,130)$ & $0.004^{b}$ & $130(125,135)$ & $120(110,125)$ & $<0.001^{b}$ & - & - & - \\
\hline High SBP (> 130 mmHg) & $24(49)$ & $76(32)$ & 0.033 & $59(62)$ & $41(22)$ & $<0.001$ & - & - & - \\
\hline $\mathrm{DBP}(\mathrm{mm} \mathrm{Hg})$ & $70(70,78)$ & $70(65,75)$ & $0.051^{b}$ & $70(70,78)$ & $70(65,75)$ & $0.011^{b}$ & - & - & - \\
\hline High DBP (> 80 mmHg) & $5(10)$ & $8(3)$ & 0.054 & $7(7)$ & $6(3)$ & 0.14 & - & - & - \\
\hline Hypoglycemia (severe episodes) & $3(6)$ & $10(4)$ & 0.48 & - & - & - & - & - & - \\
\hline Smoking & $4(9)$ & $24(11)$ & 0.80 & $6(6)$ & $22(12)$ & 0.20 & $13(10)$ & $15(10)$ & $>0.99$ \\
\hline Physical inactivity (< 1/week) & $9(19)$ & $22(10)$ & 0.084 & $10(11)$ & $21(12)$ & $>0.99$ & $11(8)$ & $20(14)$ & 0.18 \\
\hline CV complications & $5(10)$ & $5(2)$ & 0.016 & $7(7)$ & $3(2)$ & 0.018 & $9(7)$ & $1(1)$ & 0.007 \\
\hline LLD & $31(63)$ & $102(43)$ & 0.012 & $62(65)$ & $71(38)$ & $<0.001$ & - & - & - \\
\hline AHD & $22(45)$ & $73(31)$ & 0.069 & - & - & - & $62(47)$ & $33(22)$ & $<0.001$ \\
\hline MDII and OAD & $13(27)$ & $4(2)$ & $<0.001$ & - & - & - & - & - & - \\
\hline CSII & $1(2)$ & $25(10)$ & & - & - & - & - & - & - \\
\hline MD॥ & $35(71)$ & $206(88)$ & & - & - & - & - & - & - \\
\hline Reached all treatment targets & 0 & $19(8)$ & 0.052 & - & - & - & - & - & - \\
\hline
\end{tabular}

Data are $\mathrm{n}(\%)$ or median $\left(\mathrm{q}_{1}, \mathrm{q}_{3}\right)$

${ }^{a}$ Fisher's exact test unless otherwise indicated. ${ }^{b}$ Mann-Whitney $U$ test. ${ }^{c}$ Missing values for abdominal obesity/no abdominal obesity: $N=27$ $(55 \%) / 86(37 \%)$

\section{Factors associated with gender, high HbA1c, systolic} blood pressure and cardiovascular complications

Positive associations with women were found for abdominal obesity AOR 8.6 (3.9-19.0), $P<0.001$; and HDL (per mmol/l) AOR 6.1 (2.7-13.6), $P<0.001$. Negative associations with women were found for diastolic blood pressure (per mm Hg) AOR 0.91 (0.87-0.95), $P<$ 0.001; and age (per year) AOR 0.97 (0.94-0.99), $P=0$. 005. Systolic blood pressure, TC and anti-hypertensive drugs were not associated with women (all $P>0.21$ ). Nagelkerke R Square: 0.277. Hosmer and Lemeshow Test: 0.034 .

The associations with $\mathrm{HbA} 1 \mathrm{c}>70 \mathrm{mmol} / \mathrm{mol}(>8.6 \%)$ were for abdominal obesity AOR 2.7 (1.4-5.4), $P=0.004$; triglycerides (per mmol/l) AOR 1.7 (1.1-2.5), $P=0.010$; and for diastolic blood pressure (per mm Hg) AOR 1.04 (1.00-1.08), $P=0.090$. HDL, TC, age, physical inactivity, and LDL were not associated with $\mathrm{HbA1c}>70 \mathrm{mmol} /$ mol (all $P>0.16$ ). Nagelkerke R Square: 0.137. Hosmer and Lemeshow Test: 0.782 .

The B-coefficients for the associations with systolic blood pressure were for age $0.24(0.13-0.34), P<0.001$; anti-hypertensive drugs 6.5 (3.9-9.2), $P<0.001$; triglycerides (per mmol/l) 2.0 (0.4-3.6), $P=0.014$; men 4.0 (1.66.4), $P=0.001$; and for abdominal obesity 4.0 (0.8-7.3), $P=0.014$. Lipid-lowering drugs $(P=0.73)$ and diabetes duration $(P=0.99)$ were not associated with systolic blood pressure. Adjusted R Square 0.276, $P<0.001$.

Associations with cardiovascular complications were for age (per year) AOR 1.18 (1.05-1.32), $P=0.006$; 
Table 3 Associations with abdominal obesity in patients with T1DM, presented for all and gender specified

\begin{tabular}{|c|c|c|c|c|c|c|c|c|}
\hline & \multicolumn{8}{|c|}{ Abdominal obesity } \\
\hline & \multicolumn{2}{|l|}{$\begin{array}{l}\text { Both genders } \\
N=284^{a}\end{array}$} & \multicolumn{2}{|l|}{$N=272$} & \multicolumn{2}{|l|}{$\begin{array}{l}\text { Men } \\
N=158\end{array}$} & \multicolumn{2}{|l|}{$\begin{array}{l}\text { Women } \\
N=119\end{array}$} \\
\hline & $\overline{C O R}$ & $P$ & $\overline{\mathrm{AOR}}$ & $p^{b}$ & $\overline{\mathrm{AOR}}$ & $p^{b}$ & $\overline{\mathrm{AOR}}$ & $p^{b}$ \\
\hline Gender (women) & $4.5(2.3-9.0)$ & $<0.001$ & $6.5(2.9-14.5)$ & $<0.001$ & - & - & - & - \\
\hline Age (per year) & $1.02(1.00-1.05)$ & 0.11 & $1.01(0.97-1.06)$ & 0.54 & $1.03(0.97-1.10)$ & 0.31 & $1.01(0.96-1.06)$ & 0.78 \\
\hline Diabetes duration (per year) & $1.00(0.98-1.03)$ & 0.77 & - & - & - & - & - & - \\
\hline $\mathrm{HbA} 1 \mathrm{c}>70 \mathrm{mmol} / \mathrm{mol}(>8.6 \%)$ & $3.2(1.7-6.1)$ & $<0.001$ & $2.7(1.3-5.7)$ & 0.009 & $1.9(0.5-7.1)$ & 0.36 & $2.9(1.2-7.2)$ & 0.022 \\
\hline $\mathrm{TC}$ & $1.2(0.9-1.6)$ & 0.15 & - & - & - & - & - & - \\
\hline Triglycerides (per mmol/l) & $1.9(1.3-2.8)$ & $<0.001$ & $1.7(1.1-2.6)$ & 0.010 & $1.0(0.5-2.0)$ & $>0.99$ & $2.1(1.2-3.7)$ & 0.011 \\
\hline HDL (per mmol/l) & $0.7(0.3-1.6)$ & 0.34 & - & - & - & - & $0.4(0.1-1.7)$ & 0.24 \\
\hline LDL (per mmol/l) & $1.4(1.0-1.9)$ & 0.072 & $1.0(0.7-1.70)$ & 0.86 & - & - & $1.0(0.56-1.98)$ & 0.88 \\
\hline SBP (per mm Hg) & $1.04(1.01-1.07)$ & 0.004 & $1.05(1.01-1.08)$ & 0.005 & $1.01(0.94-1.10)$ & 0.72 & $1.03(0.99-1.07)$ & 0.20 \\
\hline DBP (per mm Hg) & $1.04(1.00-1.09)$ & 0.045 & $1.02(0.96-1.09)$ & 0.47 & $1.13(1.03-1.24)$ & 0.007 & - & - \\
\hline Hypoglycemia & $1.5(0.4-5.5)$ & 0.57 & - & - & - & - & - & - \\
\hline Smoking ${ }^{c}$ & $0.8(0.3-2.4)$ & 0.66 & - & - & - & - & - & - \\
\hline Physical inactivity & $2.1(0,9-4.8)$ & 0.083 & $1.7(0.5-5.2)$ & 0.36 & - & - & $3.4(0.8-14.4)$ & 0.10 \\
\hline CV complications & $5.2(1.5-18.9)$ & 0.011 & $5.7(1.1-28.9$ & 0.035 & - & - & - & - \\
\hline LLD & $2.2(1.2-4.2)$ & 0.013 & $1.9(0.9-4.1)$ & 0.096 & - & - & $3.1(1.3-7.6)$ & 0.014 \\
\hline $\mathrm{AHD}$ & $1.8(1.0-3.4)$ & 0.064 & $1.1(0.5-2.6)$ & 0.79 & $5.3(1.3-20.7)$ & 0.018 & - & - \\
\hline
\end{tabular}

${ }^{a}$ Unless indicated. ${ }^{\mathrm{b}}$ Multiple logistic regression analysis (Backward: Wald). ${ }^{\mathrm{c}}$ Missing values: $n=12$. All/men/women: Hosmer and Lemeshow: Test 0.039/0.799/ 0.471 ; Nagelkerke R Square 0.335/0.234/0.250

abdominal obesity AOR 5.5 (1.4-22.0), $P=0.017$; and for $\mathrm{LDL}$ (per mmol/l) AOR $0.3(0.1-1.1), P=0.071$. Lipid-lowering drugs, anti-hypertensive drugs and diabetes duration were not associated with cardiovascular complications (all $P>0.34$ ). Nagelkerke R Square: 0. 309. Hosmer and Lemeshow Test: 0.978.

\section{Comparisons of patients with and without CRP measurements $-A$ response analysis}

The prevalence of abdominal obesity was lower in the 171 patients with hs-CRP measurements than in the patients without hs-CRP measurements ( $13 \%$ vs $24 \%, P=0.024)$. Otherwise, they did not differ by medians for age $(P=0$. $10)$, diabetes duration $(P=0.52)$, diastolic blood pressure $(P=0.52)$, systolic blood pressure $(P=0.66)$, HDL $(P=0$. $49)$, LDL $(P=0.50)$, triglycerides $(P=0.70)$, TC $(P=0.79)$; or by prevalence of anti-hypertensive drugs $(P=0.124)$, physical inactivity $(P=0.33)$, severe hypoglycemia episodes $(P=0.38)$, HbA1c $>70 \mathrm{mmol} / \mathrm{mol} \quad(P=0.48)$, lipidlowering drugs $(P=0.72)$, cardiovascular complications $(P$ $=0.74)$, or smoking $(P=0.84)$.

\section{Discussion}

In this cross-sectional study of abdominal obesity in 284 persons with T1D, age 18-59 years, consecutively recruited from one secondary care specialist diabetes clinic, we found that cardiovascular complications, women, increasing risk levels of hs-CRP, systolic blood pressure, marked inadequate glycemic control (HbA1c > $70 \mathrm{mmol} / \mathrm{mol}$ ), and triglycerides were independently associated with abdominal obesity. Inadequate glycemic control, systolic blood pressure, increasing risk levels of hs-CRP, were in addition to abdominal obesity, also associated with triglycerides. Less patients with abdominal obesity reached the treatment targets recommended by the Swedish National Board of Health and Welfare for glycemic control (HbA1c $\leq 52 \mathrm{mmol} / \mathrm{mol}$ ) and systolic blood pressure $(\leq 130 \mathrm{mmHg})$, and no patients with abdominal obesity reached all treatment targets for TC, LDL, and blood pressure [22].

Strengths of our study are first that the population of patients with T1D was well-defined, since persons with severe comorbidities and severe substance abuse were excluded. Second, hs-CRP levels above $10 \mathrm{mg} / \mathrm{l}$ were excluded, and the CRP values were divided into 3 groups with low-, moderate- or high-risk for future cardiovascular events, as have been recommended in previous research [19]. Also, we performed a response analysis and explored whether persons with and without hs-CRP measurements differed. The patients with hs-CRP measurements had lower prevalence of abdominal obesity, otherwise they did not differ for any variable included in this study. Third, we explored interactions between the included metabolic variables. 
Table 4 Associations with low-, moderate- and high-risk hs-CRP levels

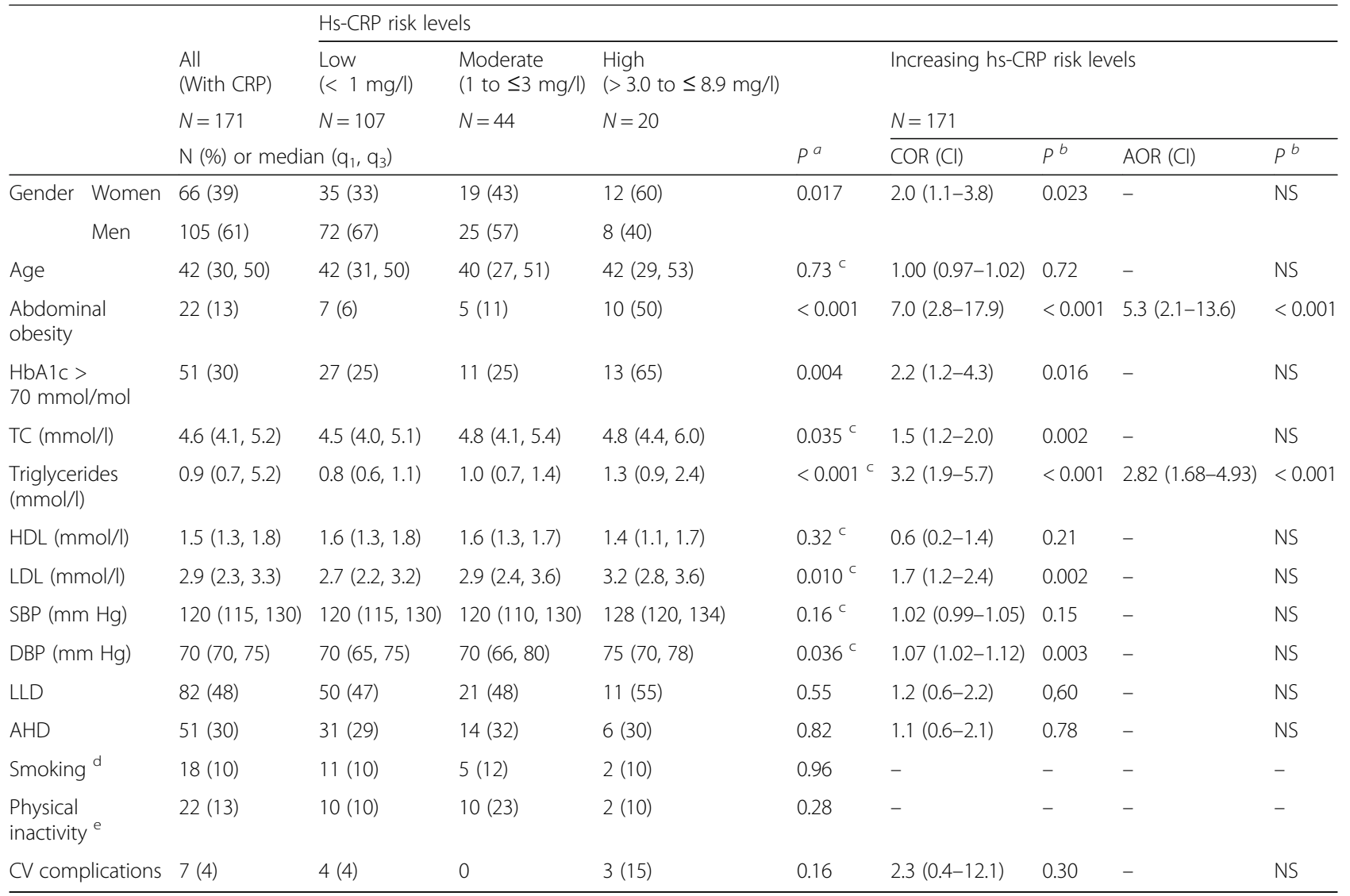

NS Non-significant

${ }^{\mathrm{a}}$ Linear-by-linear Association (Exact 2-sided) unless indicated. ${ }^{\mathrm{b}}$ Ordinal regression analyses. ${ }^{\mathrm{c}}$ Kruskal-Wallis test. Missing values: ${ }^{\mathrm{d}} n=2 ;{ }^{\mathrm{e}} n=3 .{ }^{\mathrm{d}}$ e ${ }^{\mathrm{e}}$ Not included in the ordinal regression analyses

The main limitation of our study was the rather small number of obese persons, particularly when gender sub analyses were performed. There are several possible type 2 errors. The association between the use of lipidlowering drugs and abdominal obesity did not reach significance. The prevalence of both lipid-lowering drugs and anti-hypertensive drugs in patients with cardiovascular complications was high, but the associations were not significant. Second, the number of hs-CRP values measurements was limited, as we decided not to include hs-CRP measurements stored for more than 1 year. Despite the limited number of hs-CRP measurements and the lower prevalence of obesity in persons with hs-CRP measurements, the moderate and high-risk-levels of hsCRP were strongly associated with abdominal obesity and triglyceride levels.

We have previously shown an association between alexithymia and abdominal obesity in this sample of patients with T1D [25]. In this study, we demonstrated the impact of abdominal obesity in T1D by the associations with cardiovascular complications, marked impaired glycemic control, low-grade inflammation, systolic blood pressure and triglycerides, all risk factors for future cardiovascular complications $[1,8-12,14,18]$. We found a link between impaired glycemic control and raised triglycerides, which is in accordance with findings in patients with T2D [9]. Women with T1D are at a higher risk for atherosclerosis and cardiovascular death than men $[1,2]$. One explanatory factor might be the noticeably higher prevalence of abdominal obesity in the women compared to the men with T1D, demonstrated in this study and in previous research $[5,25]$. The prevalence of general obesity was almost twice as high in the women with T1D compared to women in the general Swedish population [7]. The reasons for the excessive abdominal obesity prevalence in women with T1D were not explained by this study, and further research of this subject is suggested. Apart from abdominal obesity, the only positive association with women was higher HDL, which is not a risk factor for cardiovascular disease according to previous research [13].

Another gender difference noted was that the men had higher blood pressure than the women, which is in accordance with previous research [34]. The prevalence of high systolic blood pressure $(>130 \mathrm{mmHg})$ was significantly higher in patients using anti-hypertensive 
drugs than in non-users, and the treatment target for systolic blood pressure was not obtained for a large proportion of persons using anti-hypertensive drugs. Weight reduction might help to reduce systolic blood pressure [35]. There is evidence that low-density lipoprotein (LDL) is a causal agent in the atherothrombotic process [8]. The use of lipid-lowering drugs is associated with a lower risk for cardiovascular disease and death [14]. Patients in this study using lipid-lowering drugs were successful in reaching the treatment goals for LDL more often than non-users. Improved treatment with lipidlowering drugs is therefore suggested for patients with T1D and high LDL, in addition to weight reduction.

Due to the described detrimental effects of obesity in T1D, it is necessary to try new ways to both prevent and treat obesity. Reports of beneficial effects on weight and HbAlc have been reported for sodium-glucose cotransporter (SGLT2) inhibitors and glucagon-like peptide-1 (GLP-1) analogues [36]. Structured nutrition therapy, including reduced energy intake, lower total carbohydrate intake, and carbohydrates with lower glycemic index, has been recommended in combination with aerobic and resistance exercises [37]. As alexithymia was associated with abdominal obesity [25], psychoeducation aiming at increased emotional awareness could also be tried [28].

\section{Conclusions}

Significant associations between abdominal obesity and both cardiovascular disease and cardiovascular risk factors were found in 284 patients with T1D. Low-grade inflammation, increased systolic blood pressure, inadequate glycemic control, and increased triglycerides were linked with abdominal obesity. The obesity prevalence was particularly high in women. Action against obesity is urgent to prevent cardiovascular complications in patients withT1D.

\section{Abbreviations \\ AHD: Anti-hypertensive drugs; AOR: Adjusted odds ratio; BMl: Body mass index; Cl: Confidence interval; COR: Crude odds ratio; CSII: Continuous subcutaneous insulin infusion; GLP-1: Glucagon-like peptide-1; HDL: High- density lipoprotein; LDL: Low-density lipoprotein; LLD: Lipid-lowering drugs; MDII: Multiple daily insulin injections; OAA: Oral antidiabetic agents; SGLT2: Sodium-glucose cotransporter-2; T1D: Type 1 diabetes mellitus; TC: Total cholesterol; WC: Waist circumference}

\section{Acknowledgments}

We are grateful to Anna Lindgren, PhD, Lund University, Centre of Mathematics, Lund, Sweden, for her statistical skills.

\section{Funding}

This research was supported by the Research and Development Fund of Region Kronoberg, Växjö, Sweden, and by the Research Council of South Eastern Sweden (FORSS), Linköping, Sweden. The funding sources were not involved in the collection, analysis and interpretation of data, in the writing of the report, or in the decision to submit the article for publication.

\section{Availability of data and materials}

All data are saved at SPSS files for 10 years at the department for Research and Development, Region Kronoberg, Växjö, Sweden. The data sets are not availably publicly as individual privacy could be compromised. The data set is available from the corresponding author on reasonable request.

\section{Authors' contributions}

EOM, MT, MH, HOT, and ML-O participated as investigators and reviewed, edited, and approved of the manuscript. All authors contributed to the study design, implementation and analysis. EOM was the initiator of this study, wrote the statistical methods and the manuscript, and is the guarantor of this work and, as such, had full access to all the data in the study and takes responsibility for the integrity of the data and the accuracy of the data analysis

\section{Authors' information}

EOM is PhD, medical doctor, specialist in paediatrics and general practice. EOM works at the Department of Research and Development, and as a GP in primary care in Växjö, and is affiliated with the Department of Clinical

Sciences, Section of Endocrinology and Diabetes, Lund University, Lund. HOT is $\mathrm{PhD}$, associate professor, medical doctor, specialist in general practice, and works at the department of Research and Development, and as a GP in primary care in Växjö, and is affiliated with the Department of Clinical Sciences, Section of Family Medicine, Lund University, Malmö. Magnus Hillman is PhD and works at the Department of Clinical Sciences, the Diabetes Research Laboratory, Lund University, Lund. ML-O is PhD, medical doctor, professor at the Department of Clinical Sciences, Section of Endocrinology and Diabetes, Lund University, Lund, and works at Skane University Hospital, Lund. MT is PhD, medical doctor, specialist in internal medicine and endocrinology, and works at the department of Research and Development, and at the Central Hospital, Växjö, and is affiliated with the Department of Clinical Sciences, Section of Endocrinology and Diabetes, Lund University, Lund. All Sweden.

Ethics approval and consent to participate

The study was approved by the Regional Ethical Review Board of Linköping University, Linköping (Registration no. M120-07, T89-08). All participants provided written informed consent.

\section{Competing interests}

The authors declare that they have no competing interests.

\section{Publisher's Note}

Springer Nature remains neutral with regard to jurisdictional claims in published maps and institutional affiliations.

\section{Author details}

${ }^{1}$ Department of Clinical Sciences, Section Endocrinology and Diabetes, Lund University, Lund, Sweden. ${ }^{2}$ Department of Research and Development, Region Kronoberg, Box 1223, SE-35112 Växjö, Sweden. ${ }^{3}$ Primary Care, Region Kronoberg, Växjö, Sweden. ${ }^{4}$ Department of Clinical Sciences, Section of Family Medicine, Lund University, Malmö, Sweden. ${ }^{5}$ Department of Clinical Sciences, Diabetes Research Laboratory, Faculty of Medicine, Lund University, Lund, Sweden. 'Department of Endocrinology, Skane University Hospital, Lund, Sweden. ${ }^{7}$ Department of Internal Medicine, Central Hospital, Växjö, Sweden.

Received: 18 July 2017 Accepted: 25 April 2018

Published online: 14 May 2018

\section{References}

1. Lind M, Svensson A-M, Kosiborod M, Gudbjörnsdottir S, Pivodic A, Wedel H, et al. Glycemic control and excess mortality in type 1 diabetes. N Engl J Med. 2014:371:1972-82.

2. Colhoun HM, Rubens MB, Underwood SR, Fuller JH. The effect of type 1 diabetes mellitus on the gender difference in coronary artery calcification. J Am Coll Cardiol. 2000;36:2160-7.

3. Diabetes Control and Complications Trial Research Group. The effect of intensive treatment of diabetes on the development and progression of long-term complications in insulin-dependent diabetes mellitus. N Engl J Med. 1993;329:977-86. 
4. Purnell JQ, Hokanson JE, Cleary PA, Nathan DM, Lachin JM, Zinman B, et al. The effect of excess weight gain with intensive diabetes treatment on cardiovascular disease risk factors and atherosclerosis in type 1 diabetes: results from the diabetes control and complications trial / epidemiology of diabetes interventions and complications study (DCCT/EDIC) study. Circulation. 2013;127 https://doi.org/10.1161/CIRCULATIONAHA.111.077487.

5. Fröhlich-Reiterer EE, Rosenbauer J, Bechtold-Dalla Pozza S, Hofer SE, Schober $E$, Holl RW, et al. Predictors of increasing BMI during the course of diabetes in children and adolescents with type 1 diabetes: data from the German/ Austrian DPV multicentre survey. Arch Dis Child. 2014;99:738-43.

6. Ng M, Fleming T, Robinson M, Thomson B, Graetz N, Margono C, et al. Global, regional, and national prevalence of overweight and obesity in children and adults during 1980-2013: a systematic analysis for the global burden of disease study 2013. Lancet. 2014;384:766-81.

7. SCB. Undersökningarna av levnadsförhållanden år 2009. [internet]. 2017 Apr. Available from: www.scb.se/ulf.

8. Ridker PM. LDL cholesterol: controversies and future therapeutic directions. Lancet. 2014;384:607-17.

9. Nordestgaard BG. Triglyceride-rich lipoproteins and atherosclerotic cardiovascular disease. Circ Res. 2016;118:547-63.

10. Nordestgaard BG, Varbo A. Triglycerides and cardiovascular disease. Lancet. 2014;384:626-35

11. Langsted A, Freiberg JJ, Tybjaerg-Hansen A, Schnohr P, Jensen GB, Nordestgaard BG. Nonfasting cholesterol and triglycerides and association with risk of myocardial infarction and total mortality: the Copenhagen City heart study with 31 years of follow-up. J Intern Med. 2011;270:65-75.

12. Bjornstad P, Maahs DM, Wadwa RP, Pyle L, Rewers M, Eckel RH, et al. Plasma triglycerides predict incident albuminuria and progression of coronary artery calcification in adults with type 1 diabetes: the coronary artery calcification in type 1 diabetes study. J Clin Lipidol. 2014;8:576-83.

13. Rader DJ, Hovingh GK. HDL and cardiovascular disease. Lancet. 2014;384: 618-25

14. Hero C, Rawshani A, Svensson A-M, Franzén S, Eliasson B, Eeg-Olofsson K, et al. Association between use of lipid-lowering therapy and cardiovascular diseases and death in individuals with type 1 diabetes. Diabetes Care. 2016;39:9961003.

15. Wellen KE, Hotamisligil GS. Inflammation, stress, and diabetes. J Clin Invest. 2005:115:1111-9.

16. Saito M, Ishimitsu T, Minami J, Ono H, Ohrui M, Matsuoka H. Relations of plasma high-sensitivity C-reactive protein to traditional cardiovascular risk factors. Atherosclerosis. 2003;167:73-9.

17. Esser N, Legrand-Poels S, Piette J, Scheen AJ, Paquot N. Inflammation as a link between obesity, metabolic syndrome and type 2 diabetes. Diabetes Res Clin Pract. 2014;105:141-50.

18. De Ferranti SD, De Boer IH, Fonseca V, Fox CS, Golden SH, Lavie CJ, et al. Type 1 diabetes mellitus and cardiovascular disease. Circulation. 2014;130: 1110-30.

19. Ridker PM. Clinical application of C-reactive protein for cardiovascular disease detection and prevention. Circulation. 2003:107:363-9.

20. Mold C, Gewurz H, Du Clos TW. Regulation of complement activation by C-reactive protein. Immunopharmacology. 1999:42:23-30.

21. Casagrande SS, Fradkin JE, Saydah SH, Rust KF, Cowie CC. The prevalence of meeting A1C, blood pressure, and LDL goals among people with diabetes, 1988-2010. Diabetes Care. 2013;36:2271-9.

22. The National Board of Health and Welfare. Swedish National Guidelines for diabetes. 2009

23. Eeg-Olofsson K, Cederholm J, Nilsson PM, Gudbjörnsdóttir S, Eliasson B. Glycemic and risk factor control in type 1 diabetes. Diabetes Care. 2007;30: 496-502.

24. Chobanian AV, Bakris GL, Black HR, Cushman WC, Green LA, Izzo Jr JL, et al. The seventh report of the joint national committee on prevention, detection, evaluation, and treatment of high blood pressure: the JNC 7 report. JAMA. 2003;289:2560-71.

25. Melin EO, Svensson R, Thunander M, Hillman M, Thulesius HO, LandinOlsson M. Gender, alexithymia and physical inactivity associated with abdominal obesity in type 1 diabetes mellitus: a cross sectional study at a secondary care hospital diabetes clinic. BMC Obes. 2017:4:21.

26. Melin EO, Thunander M, Svensson R, Landin-Olsson M, Thulesius HO Depression, obesity and smoking were independently associated with inadequate glycemic control in patients with type 1 diabetes. Eur J Endocrinol. 2013;168:861-9.
27. Melin EO, Thunander M, Landin-Olsson M, Hillman M, Thulesius HO Depression, smoking, physical inactivity and season independently associated with midnight salivary cortisol in type 1 diabetes. BMC Endocr Disord. 2014:14:75.

28. Melin EO, Svensson R, Gustavsson S- $\AA$, Winberg A, Denward-Olah E, Landin-Olsson $\mathrm{M}$, et al. Affect school and script analysis versus basic body awareness therapy in the treatment of psychological symptoms in patients with diabetes and high HbA1c concentrations: two study protocols for two randomized controlled trials. Trials. 2016;17:221.

29. Melin E. Thesis. Psychosomatic aspects on diabetes and chronic pain alexithymia, depression and salivary cortisol the affect school and script analysis therapy. Lund: Lund University; 2014.

30. Klein S, Allison DB, Heymsfield SB, Kelley DE, Leibel RL, Nonas C, et al. Waist circumference and Cardiometabolic risk: a consensus statement from shaping America's health: Association for Weight Management and Obesity Prevention; NAASO, the Obesity Society; the American Society for Nutrition; and the American Diabetes Association. Obesity. 2007;15:1061-7.

31. Tanamas SK, Permatahati V, Ng WL, Backholer K, Wolfe R, Shaw JE, et al. Estimating the proportion of metabolic health outcomes attributable to obesity: a cross-sectional exploration of body mass index and waist circumference combinations. BMC Obes. 2016;3:4.

32. Jacobsen BK, Aars NA. Changes in waist circumference and the prevalence of abdominal obesity during 1994-2008 - cross-sectional and longitudinal results from two surveys: the Tromsø study. BMC Obes. 2016:3:41.

33. Ford ES, Giles WH, Dietz WH. Prevalence of the metabolic syndrome among US adults: findings from the third National Health and nutrition examination survey. JAMA. 2002;287:356-9.

34. Maranon R, Reckelhoff JF. Sex and gender differences in control of blood pressure. Clin Sci. 2013;125:311-8.

35. Neter JE, Stam BE, Kok FJ, Grobbee DE, Geleijnse JM. Influence of weight reduction on blood pressure. Hypertension. 2003:42:878-84.

36. Bode BW, Garg SK. The emerging role of adjunctive noninsulin antihyperglycemic therapy in the management of type 1 diabetes. Endocr Pract. 2015;22:220-30.

37. Mottalib A, Kasetty M, Mar JY, Elseaidy T, Ashrafzadeh S, Hamdy O. Weight Management in Patients with type 1 diabetes and obesity. Curr Diab Rep. 2017;17:92.

\section{Ready to submit your research? Choose BMC and benefit from:}

- fast, convenient online submission

- thorough peer review by experienced researchers in your field

- rapid publication on acceptance

- support for research data, including large and complex data types

- gold Open Access which fosters wider collaboration and increased citations

- maximum visibility for your research: over $100 \mathrm{M}$ website views per year

At BMC, research is always in progress.

Learn more biomedcentral.com/submissions 